\title{
Postdrug retention of diazepam's effects on habituation to a novel environment in an animal model of anxiety
}

\author{
HARALD K. TAUKULIS and RALPH W. MCKAY \\ University of New Brunswick, Saint John, New Brunswick, Canada
}

\begin{abstract}
In two experiments it was demonstrated that diazepam can have habituation-facilitating effects that will be retained beyond the termination of drug action. Rats given multiple exposures to a novel plus maze $30 \mathrm{~min}$ after an injection of diazepam $(2.0 \mathrm{mg} / \mathrm{kg})$ on each occasion explored the "open" (exposed) arms of the maze more than animals injected with saline or yohimbine hydrochloride did. All groups exhibited equivalent rates of increase in this activity over repeated trials in the maze-behavior that was interpreted to reflect habituation to the environment and, in particular, habituation to the somewhat aversive open arms. When shifted to saline injections in later trials, the animals with diazepam experience retained their relatively higher level of open-arm exploration for as long as 56 days after their last diazepam injection. In a third experiment, animals were confined to the open arms of the maze on five occasions following either a diazepam or a saline injection and were then tested drug-free in the complete maze on subsequent trials. Here the diazepam-experienced animals did not differ from the saline controls. The forced exposure to the open arms, although unequally stressful to the diazepam- and saline-injected animals as indicated by their different fecal bolus counts, yielded comparable, high levels of openarm exploration in both groups of subjects once they were permitted access to the entire maze.
\end{abstract}

Some animal models that are used to study the anxiolytic properties of drugs like the benzodiazepines rely on the natural tendency of rodents to behave cautiously when encountering novel stimuli or situations (Crawley, 1985; File, 1985). Anxiolytic agents tend to reduce this cautiousness in some systematic fashion, and in a good model, this change in behavior should correlate well with anxiety reduction in humans. In both human and animal subjects, repeated exposure to novel, arousing stimuli can result in habituation, defined as diminished behavioral and/or physiological responses when encounters are repeated. If an anxiolytic drug is administered during the habituation process, the drug may facilitate, interfere with, or have no effect on the rate at which habituation develops and/or the degree to which it is retained once the subject is drug-free.

Anxiolytic drugs of the benzodiazepine class have been shown to affect habituation to a novel stimulus or situation in different ways, depending upon the details of the experiment. In some studies, facilitation of habituation by chlordiazepoxide has been reported. In rats, Iwahara and Sakama (1972) found that this drug accelerated a diminution of ambulation within an open field across four trials, while rearing, defecation, and urination measures

This research was supported in part by Grant 0002027 from the Natural Sciences and Engineering Research Council of Canada. The authors wish to thank Ann Chapman and Monica Anderson for their assistance with various aspects of this work. Correspondence concerning this article should be addressed to H. K. Taukulis, Florence M. Christie Laboratory for Life Sciences, University of New Brunswick, Saint John, New Brunswick, Canada E2L 4L5. remained unaffected. Figler (1973) observed attenuated threat displays in Siamese fighting fish treated with chlordiazepoxide and an accelerated reduction of this behavior across three observations made within a single trial. In human subjects, too, the drug has been reported to have facilitatory effects when galvanic skin responses to auditory stimuli have been measured in patients suffering from anxiety states (Lader \& Wing, 1965).

Retention of the habituation effect beyond the period of benzodiazepine administration has also been described. Figler (1973), in his study of Siamese fighting fish, observed that chlordiazepoxide-induced reductions in threat displays transferred to a second test performed $24 \mathrm{~h}$ after drug exposure. In this study, however, traces of the drug or its active metabolite may have remained in the animals' bodies and may have contributed to the persistence of habituation.

Lister and File (1987) tested mice 2 weeks after a single chlordiazepoxide injection and found that the drug had interfered with rather than facilitated habituation. They placed mice onto a holeboard apparatus that the animals explored by dipping their noses into the apertures in the floor of the device. This behavior occurred with reduced frequency during a second exposure, but this reduction was attenuated in mice that had received chlordiazepoxide prior to their first exposure, an effect that did not appear to be attributable to chlordiazepoxide-induced amnesia or state-dependent retrieval. Platel and Porsolt (1982) also found a chlordiazepoxide-induced impairment of habituation in mice given two sessions, spaced 1-7 days apart, in an activity chamber. Unlike control animals given 
no drug, the animals injected with chlordiazepoxide immediately after the first session showed no decrease in activity level in the second session.

File and Pellow (1988) predicted that benzodiazepine receptor inverse agonists, which are substances that often induce effects in the opposite direction to those of receptor agonists like chlordiazepoxide, might facilitate habituation in the holeboard apparatus. However, two such agents, $\mathrm{N}$-methyl- $\beta$-carboline-3-carboxamide (FG 7142) and methyl 6,7-dimethoxy-4-ethyl- $\beta$-carboline-3-carboxylate (DMCM) were shown to be without effects in rats. In contrast, Venault et al. (1986) reported that mice injected with low doses of methyl- $\beta$-carboline-3-carboxylate $(\beta$-CCM) increased their consumption of familiar food (a putative index of habituation to novelty) during their second exposure to an unfamiliar cage.

Because the evidence for the effects of benzodiazepines on the development and retention of habituation has been equivocal, we sought to explore this issue further. The present experiments were designed to address this problem through the use of an animal model of anxiety that has proven to be a reasonably reliable screening device for many anxiolytic agents: an elevated plus maze (Pellow, 1986; Pellow \& File, 1986). Exposure to this apparatus appears to be mildly stressful to a rat, and an animal's behavior herein is known to be affected by substances that have anxiolytic effects in humans (Lister, 1987; Pellow, Chopin, File, \& Briley, 1985; Taukulis \& Brake, 1988, 1989). This type of maze is, essentially, comprised of two "open arms" (narrow, exposed boards) and two "enclosed arms" (surrounded by high walls). The amount of time rats and mice will spend exploring the open arms (the nonpreferred areas) will be increased by a variety of anxiolytics, including diazepam. The aversiveness of the open arms is inferred from Pellow et al.'s (1985) observations that, when animals confined to only the open arms were compared with those confined to the enclosed arms, the former spent more time motionless, produced more fecal boli, and had higher plasma corticosterone levels. "Habituation"' to this maze, indexed by a gradually increasing tendency to enter the open arms, is presumed to reflect a diminishing aversiveness of these areas.

\section{EXPERIMENT 1}

In the first experiment, rats were given five exposures to the plus maze while either drug-free or after an injection of the benzodiazepine diazepam or the alpha 2-adrenoreceptor antagonist yohimbine hydrochloride. Yohimbine, a drug reported to have anxiogenic effects in human subjects, will decrease open-arm activity in the plus maze in rats (File \& Johnston, 1987; Johnston, 1988; Johnston $\&$ File, 1989; Pellow et al., 1985). It was included here to provide a contrast to the anxiolytic effects of diazepam and to validate the efficacy of our version of the plusmaze technique, which differed somewhat from that described by others (e.g., Lister, 1987; Nomikos \& Spyraki, 1988; Pellow et al., 1985). Following the five habitua- tion trials, five drug-free tests were performed on all the animals in order to determine if the patterns of behavior established in the drug states would be retained.

\section{Method}

Subjects. The animals used were male Long-Evans rats obtained from Charles River Canada, St. Constant, Quebec at a weight range of $225-250 \mathrm{~g}$; they were maintained in the laboratory until they had reached an average weight of $330 \mathrm{~g}$. The animals were individually housed in suspended steel cages and were provided with food (Purina Rat Chow) and water ad lib. The animal holding room was maintained at $22-23^{\circ} \mathrm{C}$ with a $14: 10$-h light:dark cycle.

Apparatus. The black-painted plus maze consisted of two opposing open arms $(50 \times 11 \mathrm{~cm})$ and two opposing enclosed arms $(50 \times 11 \mathrm{~cm})$ surrounded by plywood walls $(40 \mathrm{~cm}$ high). It was elevated to a height of $84 \mathrm{~cm}$ above the floor of the room in which it was housed. An $11-\mathrm{cm}$ square at the juncture of the four arms was the start area for each animal during all trials. An open field $(66.0 \times 72.4 \mathrm{~cm})$ was used as the preliminary holding area; each subject was placed there for $5 \mathrm{~min}$ before its trial in the plus maze in order to increase maze activity levels (Pellow et al., 1985). Behavior was recorded with an 8-mm Sony video camera; videotapes were subsequently scored by an experimenter who was unaware of the group membership of each animal.

Drugs. All substances were administered intraperitoneally. The drugs used were diazepam (Roche injectable) in a $5-\mathrm{mg} / \mathrm{ml}$ solution at a dose of $2.0 \mathrm{mg} / \mathrm{kg}$, and yohimbine hydrochloride (Sigma) in a $1.25-\mathrm{ml} / \mathrm{kg}$ solution with distilled water at a dose of $2.5 \mathrm{mg} / \mathrm{kg}$. These specific doses were chosen because they were previously shown to reliably induce shifts toward greater or lesser open-arm activity during single exposures to the maze. In the case of diazepam, it was known that this dose would not cause significant ataxia or alterations in nonspecific activity in this particular apparatus. Saline injections, when administered, were equivalent by volume to the drug injections.

Procedure. The animals were weighed and assigned to one of three groups ( $n=10$ in each case): saline control (S-S), diazepam (D-S), and yohimbine (Y-S). All animals received five habituation trials spaced $48 \mathrm{~h}$ apart. On each trial, an animal was given an intraperitoneal injection of drug or saline appropriate to its group designation (indicated by the first letter of its group name) and then replaced in its home cage. Twenty-five minutes thereafter, it was placed into the open field for $5 \mathrm{~min}$ and then into the plus maze for an additional $5 \mathrm{~min}$. Plus-maze placement occurred in the center square at the juncture of the four arms with the animal's body oriented toward one of the open arms. On the rare occasion when an animal fell from one of the open arms, timing stopped until it had been replaced on the portion of the arm from which it had fallen.

During Trials 6-10, the same procedure was followed, except that saline injections (indicated by the " $S$ "' in each group name) were administered to all the animals $30 \mathrm{~min}$ prior to their exposure to the plus maze. These trials were spaced $120 \mathrm{~h}$ apart, except for Trial 10, which occurred 38 days after Trial 9.

The measures that were subsequently derived from the videotapes were the number of entries into the open and enclosed arms and the amount of time spent in each type of arm. An arm entry was counted when all four of an animal's paws crossed the entrance boundary of an arm immediately following a period during which none of its paws were within that boundary. Similarly, time spent in an arm was defined as the period during which all four paws were within the arm's entrance boundary.

\section{Results}

The mean percentage of time spent in the open arms of the plus maze by each group is shown in Figure 1 (upper 

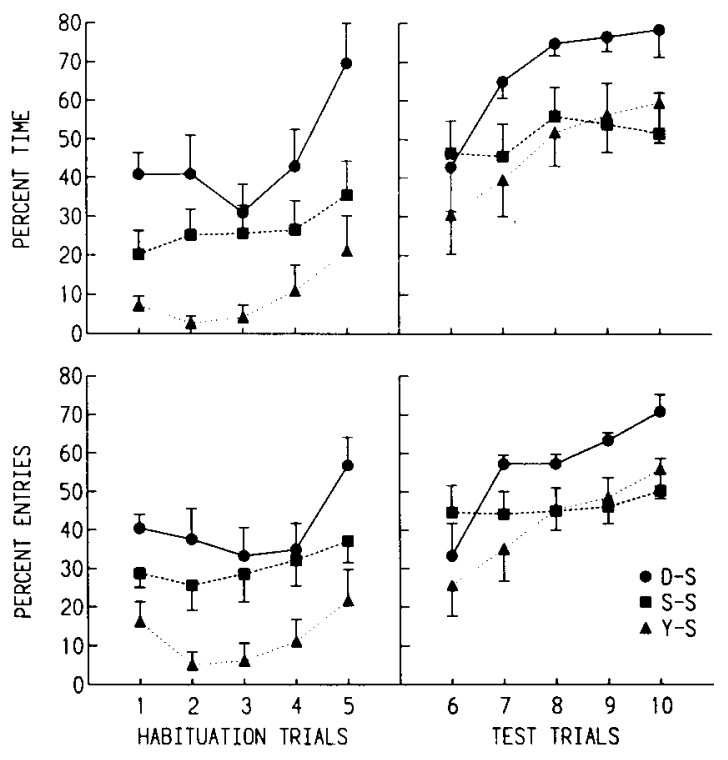

Figure 1. Mean percentages $( \pm S E M)$ of time spent in the open arms of the plus maze (upper graph) and entries into these arms (lower graph) by the three groups in Experiment 1. For Trials 1-5 (habituation), the rats were injected with either diazepam (Group D-S), saline (Group S-S), or yohimbine (Group Y-S); for Trials 6-10 (test), all animals received injections of isotonic saline.

graph). The left portion of the graph shows the results of the five habituation trials during which Groups D-S, S-S, and $\mathrm{Y}-\mathrm{S}$ received diazepam, saline, or yohimbine, respectively. An analysis of variance (ANOVA; $3 \times 5$, groups $\times$ trials) indicated that the three groups differed significantly on this measure $[F(2,27)=9.54, p<.001]$. These percentages increased across trials $[F(4,108)=$ $9.59, p<.001]$. Planned pairwise comparisons $(2 \times 5$ ANOVAs, groups $\times$ trials) revealed that Group D-S spent more time in the open arms than Group S-S did $[F(1,27)=5.03, p<.05]$, and Group Y-S spent less time in the open arms than the saline group did $[F(1,27)=$ $4.51, p<.05]$. The groups did not differ in terms of the rate at which their exploration of the open arms increased across trials, as indicated by the fact that the overall ANOVA yielded no groups $\times$ trials interaction $[F(8,108)$ $=1.34, p>.20]$.

The mean percentage of entries into the open arms for each group across the five habituation trials is shown in the lower graph in Figure 1. The analysis of these percentages was similar in most respects to that just described for the percent-time data. A $3 \times 5$ ANOVA yielded a significant difference among the groups $[F(2,27)=9.77$, $p<.001]$, and the animals as a whole demonstrated a significant habituation effect across trials $[F(4,108)=$ $6.19, p<.001]$. Once again, the groups $\times$ trials interaction did not attain significance. Groups D-S and Y-S were compared with Group S-S by means of $2 \times 5$ ANOVAs. These yielded a significant difference between Groups S-S and Y-S $[F(1,27)=7.91, p<.01]$, but not between Groups D-S and S-S $[F(1,27)=2.39, p>$ $.10]$. It is not unusual, we have found, to obtain a difference between groups with the time measure and not with the entries measure, and we believe that the latter is somewhat less reliable. In part, this derives from the fact that the numbers on which the percentages are based are small, and this tends to translate into greater variability than is true with the time measure.

The right portions of the graphs in Figure 1 show the results of the five posthabituation trials $(6-10)$ during which all the animals had received saline injections only. Due to heterogeneity of variance among groups, MannWhitney $U$ tests were performed in which Groups D-S and $\mathrm{Y}-\mathrm{S}$ were separately compared with Group S-S on each trial. These comparisons revealed that there were no differences in percentage of time spent in the open arms between Groups D-S and S-S in Trial 6. However, Group D-S spent a greater proportion of time in the open arms and entered them proportionately more frequently during Trials 7,8 , and 10 ( $p<.05$ in all cases). The results of Trial 9 were similar but did not reach significance. Comparisons between Groups S-S and Y-S revealed no differences in percent time in open arms or in percent entries on any of the five posthabituation trials.

In any analysis of plus-maze behavior, the total number of arm entries is usually examined as an index of general activity. Figure 2 depicts the group means obtained for this measure. A $3 \times 5$ ANOVA revealed a significant decrease in activity across Trials $1-5[F(4,108)=13.73$, $p<.001]$. Although there was no overall groups effect $[F(2,27)=1.93, p>.10]$, a significant groups $\times$ trials interaction was evident $[F(8,108)=2.75, p<.01]$, reflecting predominantly the greater activity shown by Group D-S relative to Group Y-S on Trials 1 and 5. A similar analysis of the total entries was performed for Trials 6-9. The increase in total entries across trials was significant for the animals as a whole $[F(3,81)=5.83$, $p<.01]$, but neither a groups effect nor a groups $\times$ trials interaction appeared $(p s>.30)$. Trial 10 was analyzed separately because the temporal gap that separated it from Trial 9 was much greater than the gaps between pairs of

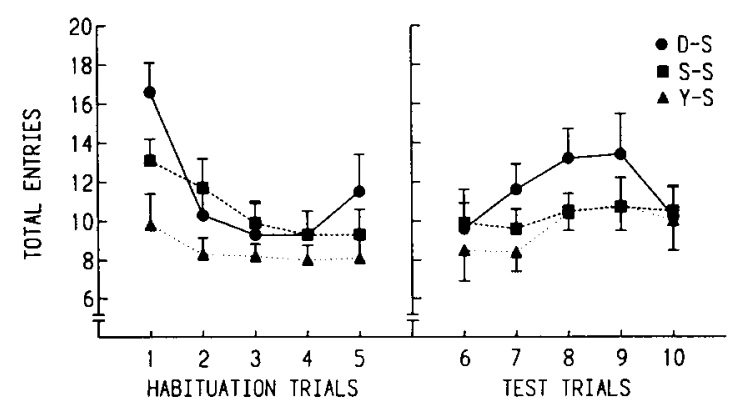

Figure 2. Mean number $( \pm S E M)$ of entries into the open and enclosed arms combined, made by the three groups in Experiment 1 during the five habituation and five saline test trials. 
the other four test trials; this may have been responsible for the dip in the curve for Group D-S seen between Trials 9 and 10 in Figure 2. The group means on this day were statistically identical $[F(2,27)<1]$.

\section{Discussion}

The previously reported effects of diazepam and yohimbine on activity in the plus maze were replicated: the drugs increased and decreased open-arm exploration, respectively. These findings attested to the efficacy of our procedures. However, no differences in the rate of habituation (i.e., the increments in open-arm activity from one trial to the next) were detected. Although Group D-S showed a marked upward shift from Trial 4 to Trial 5 that may have been the beginning of a trend, it is equally likely that this jump represented normal variability in the effects of this drug. We tentatively concluded, therefore, that diazepam neither facilitates nor retards the rate of habituation when administered to rats in this preparation.

For Trials 6-10, all the animals were shifted to saline injections and tested in a drug-free state. Groups $\mathrm{S}-\mathrm{S}$ and Y-S continued to exhibit a slow upward trend toward more open-arm exploration. Group D-S, after an initial decline in Trial 6, spent a greater amount of time in the open arms than either of the other groups did, thereby exhibiting a retention of the drug's effects on exploration beyond the period of drug administration. This could not have been due to the incomplete metabolism of diazepam, particularly since the effect was still evident on Trial 10 , which occurred 56 days after the last diazepam injection. This effect is particularly interesting because of the wellknown capacity of diazepam to induce anterograde amnesia in a variety of experimental paradigms (Thiebot, 1985). If the drug had caused amnesia for the first five plus-maze experiences, then a diminution in open-arm activity should have been seen during the latter five trials. Perhaps partial amnesia did occur, as suggested by the dip in percent time and entries seen on Trial 6. The animals' experience on this trial may have served as a "reminder" which triggered the increases evident in Trials 710. Alternatively, the Trial 6 dip may have reflected an instance of partial state-dependent retrieval (Overton, 1964 ) in which information acquired under the influence of a drug is not completely recoverable in the drug-free state. Regardless of which explanation is tenable here, it is clear that the loss of habituation was transient.

When overall activity in the plus maze as measured by total arm entries was examined, a different pattern of habituation was observed. All the animals explored the maze the most during their first exposure to it on Trial 1; group differences were observable, with Group D-S entering the greatest number of arms and Group Y-S the least. Thereafter, exploration declined and stabilized, and no group differences were evident. Group Y-S did, however, make fewer entries than Group S-S did (though this did not reach statistical significance), a finding that is in agreement with other reports of yohimbine's actions (File
\& Johnston, 1987; Johnston \& File, 1989). When the shift was made from drugs to saline in Trials 6-10, Groups D-S and Y-S exhibited a slight tendency to increase exploration, but statistical differences among groups were not obtained.

\section{EXPERIMENT 2}

In Experiment 1, the effects of diazepam on the first five trials were retained when the animals were shifted to saline in Trials 6-10. The experiment did not, however, include a group that continued to get diazepam in the latter trials. This would have allowed us to compare the behavior of shifted with nonshifted groups. It is possible that, even if such a group had been included, no difference would have been detected, because the animals in Group D-S were already showing a ceiling effect (i.e., exhibiting maximal percentages of open-arm activity that could not go much higher). Experiment 2 was designed to avoid these problems. It was essentially a replication of Experiment 1, except that each animal experienced only two habituation trials preceded by either a diazepam or a saline injection. Two test trials followed in which an animal continued to receive the same substance or was shifted to the other one. The reduced number of trials was expected to insure a relatively low degree of habituation, thereby avoiding a ceiling effect and allowing a shifted versus nonshifted comparison to be made.

\section{Method}

Subjects and Apparatus. Thirty-two male Long-Evans rats were used as subjects. They were obtained from the supplier at 225-250 g and maintained in the laboratory until they weighed an average of $331 \mathrm{~g}$ at the beginning of the experiment. In all unspecified respects they were treated exactly like the animals in Experiment 1.

The equipment employed was identical to that used in the previous study.

Procedure. Each plus-maze trial was identical to that described in Experiment 1. Half of the animals $(n=16)$ received diazepam $(2.0 \mathrm{mg} / \mathrm{kg}) 25 \mathrm{~min}$ prior to a 5 -min placement into an open field, followed by a 5 -min exposure to the maze. The remaining animals received saline $(2.0 \mathrm{ml} / \mathrm{kg})$ injections. Trials 1 and 2 occurred 10 days apart. For Trials 3 and 4 , the animals with diazepam experience were split into two groups, matched on the basis of their plus-maze performance during the second habituation trial. Half of these (Group D-D, $n=8$ ) continued to receive diazepam, and the rest (Group D-S) were shifted to saline injections. (Due to error during Trial 3, 1 animal from Group D-D had to be deleted from the experiment, reducing this group to 7 subjects.) Similarly, half of the animals with saline experience continued to receive saline injections (Group S-S), and half were shifted to diazepam (Group S-D). Trials 3 and 4 occurred at 10 and 17 days, respectively, after Trial 2 .

\section{Results and Discussion}

The percentage of time spent in the open arms in Trials 1-4 and the percentage of entries are illustrated in Figure 3. The left half of each graph shows that diazepam had its expected effect in Trials 1 and 2. The diazepam animals exhibited greater open-arm exploration both in terms of time $[F(1,30)=8.79, p<.01]$ and entries 

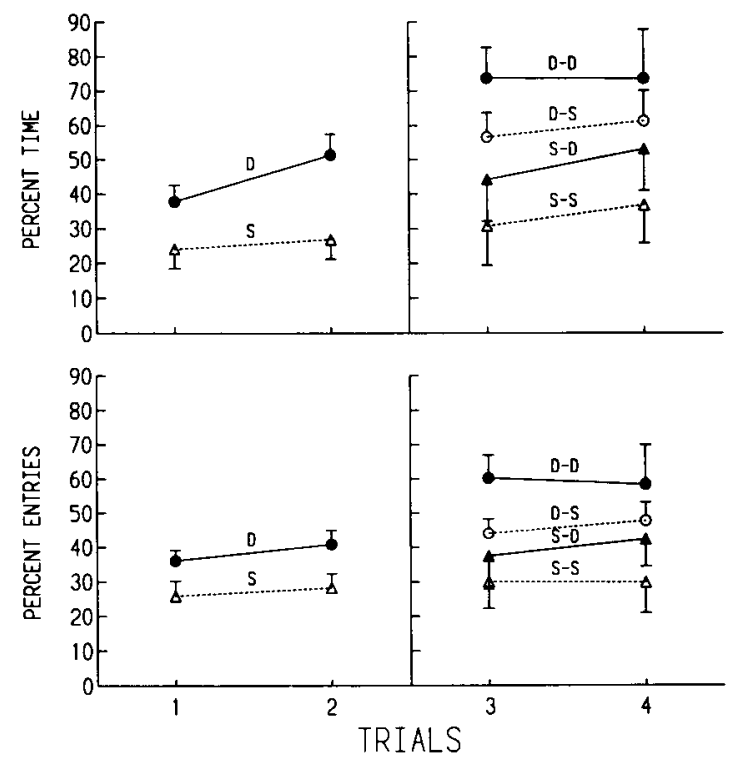

Figure 3. Mean percentages $( \pm S E M)$ of time spent in the open arms of the plus maze (upper graph) and entries into these arms (lower graph) shown by the groups in Experiment 2. For Trials 1 and 2, two sets of animals received injections of either diazepam (D) or saline (S). For Trials 3 and 4, half of these animals continued to receive injections of the same substances (Groups D-D and S-S), while half were switched to the opposite substance (Groups D-S and S-D).

$[F(1,30)=7.33, p<.02]$. Neither a trials effect nor a groups $\times$ trials interaction was noted for either measure. An analysis of total entries also yielded no differences for either the groups or trials factor.

The right half of each graph shows that the animals with diazepam experience during Trials 1 and 2 exhibited greater open-arm activity during Trials 3 and 4 than those with saline experience did, both when they continued to receive the drug (Group D-D) and when they did not (Group D-S). A $2 \times 2 \times 2$ (drug experience $\times$ test injection $\times$ trials) ANOVA yielded significance for the drug experience factor on both the time $[F(1,27)=4.55, p<$ $.05]$ and entries $[F(1,27)=4.64, p<.05]$ measures. A comparison of Groups D-S and S-S showed that the retention effect of Experiment 1 was replicated: the enhanced open-arm activity induced by diazepam persisted even after the drug was withdrawn.

The test injection (i.e., the substance injected immediately prior to Trials 3 and 4) factor in the ANOVA did not reach significance for either measure, nor was there a significant interaction with the drug experience factor. This means that Group D-D did not differ statistically from Group D-S, and Group S-D did not differ from Group S-S. However, the differences between pairs were in the predicted direction, with the two groups that received diazepam during Trials 3 and 4 exhibiting greater open-arm activity than the two groups that were drug-free did. No difference between trials was observed for percent time $[F(1,27)=2.26, p>.01]$ or percent entries
$[F(1,27)<1]$, nor was an interaction of any type detected among factors. A further ANOVA analyzing differences in total entries for Trials 3 and 4 yielded no significant effects.

As in Experiment 1, it is unlikely that the retention effect shown by Group D-S was attributable to persisting traces of diazepam or its metabolites. Diazepam was administered only twice before saline tests were carried out, at 10 and 17 days after the last drug administration, so an accumulation of active substances was improbable.

\section{EXPERIMENT 3}

In Experiments 1 and 2, the animals were allowed to freely explore all portions of the maze, and diazepam facilitated exploration of the more aversive open arms, an effect that persisted into the drug-free state. How critical is it that the exploration be voluntary and gradual? In a procedure called flooding or response prevention, human or animal subjects are abruptly (and, in the case of animals, involuntarily) exposed to an aversive stimulus or situation in an effort to extinguish avoidance behavior (e.g., Baum, 1970). Will diazepam facilitate the process of learning that a stimulus or situation is harmless under such conditions, or will it retard this process? Most animal studies examining this question have used some type of conditioned avoidance paradigm and have generally concluded that benzodiazepines will either have no effect on the extinction process or will retard it (Gorman, Dyak, \& Reid, 1979; Kamano, 1972; Thyer, Baum, \& Reid, 1988). Experiment 3 was designed to address this question in the context of the present paradigm. During five habituation trials, animals were confined to only open (relatively more aversive) arms of the plus-maze after either an injection of diazepam or an injection of inert saline. Subsequently, their behavior in the total maze was examined.

\section{Method}

Subjects. The subjects were 24 Long-Evans male rats obtained from Charles River Canada at a weight range of 75-100 g. They were raised until they had attained a mean body weight of $348 \mathrm{~g}$ immediately prior to the beginning of the experiment and were housed and maintained under the same conditions described in Experiment 1

Apparatus. The equipment used was the same as that used in Experiment 1, except that the entrance to each enclosed arm of the plus maze was, at times, blocked with black posterboard.

Procedure. Over a 15-day period, all the animals were given five 5-min exposures to the open arms of the plus maze. This was preceded $30 \mathrm{~min}$ earlier by either a $2.0-\mathrm{mg} / \mathrm{kg}$ injection (i.p.) of diazepam (Group D-S, $n=12$ ) or an equivalent-by-volume injection of physiological saline (Group $S-S, n=12$ ). Activity was not videotaped, but fecal boli were counted following each trial. In order to give all the animals equal experience with the diazepam, those that had received saline in the maze received a $2.0-\mathrm{mg} / \mathrm{kg}$ diazepam injection either $24 \mathrm{~h}$ before $(n=6)$ or $24 \mathrm{~h}$ after $(n=6)$ their exposure to the maze. For this procedure, they were simply removed from their home cages, injected, and replaced. Similarly, the animals that had received diazepam in the maze received an equal number of saline injections in their home cages. 
In the next phase of this experiment, all the animals were tested three times in the plus maze with the barriers to the enclosed arms removed. Test 1 occurred 48-72 h following their last exposure to the open arms, Test 2 occurred at $96 \mathrm{~h}$ after Test 1 , and Test 3 occurred 28 days after Test 2 . For each test, an animal was injected with normal saline, placed into the open field $25 \mathrm{~min}$ thereafter for a 5 -min period, and then set into the plus maze for $5 \mathrm{~min}$, where its behavior was videotaped.

\section{Results and Discussion}

The mean bolus counts obtained for both groups during the five open-arm habituation trials of this experiment are shown in Figure 4. Higher counts were obtained for Group S-S than for Group D-S $[F(1,22)=9.65, p<$ $.005]$, suggesting that the open arms were less stressful for the latter group. This difference had not diminished by Trial 5 , as indicated by the absence of a groups $\times$ trials interaction $[F(4,88)<1]$.

Mean percentages of time spent in the open arms and entries into these arms during the three full-maze test trials are shown in Figure 5. Two $2 \times 3$ (groups $\times$ tests) ANOVAs revealed no differences between groups and no interaction between factors on either measure (all ps $>.10$ ). However, both percent time and percent entries were found to increase markedly across trials in both groups (both $p \mathrm{~s}<.001$ ). At no time did the groups differ in their total number of arm entries, nor did this measure differ among tests (all $p s>.10$ ).

The percentage of open-arm activity shown by both groups during the full-maze test trials was high. Note that the percentages of time spent in the open arms on Tests 2 and 3 were comparable to those recorded for Group D-S during the last four posthabituation trials (Trials 7-10) of Experiment 1 (see Figure 1) and also to the percentages obtained for Group D-S during Trials 3 and 4 of Experiment 2 . These similarities suggest that forced exposure to the open arms did cause increased full-maze exploration. However, in the absence of control groups that were not exposed to the open arms during the first phase, this conclusion must remain tentative. Nonetheless, it appears that a diazepam-induced reduction in stress during the

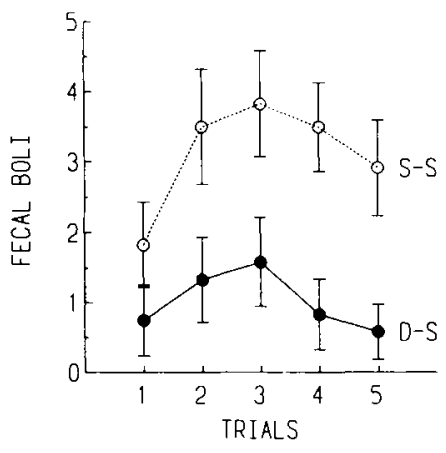

Figure 4. Mean numbers $( \pm S E M)$ of fecal boli collected following each of five exposures to the open arms of the plus maze in Experiment 3. Prior to each of these trials, the rats were injected with either diazepam (Group D-S) or saline (S-S).
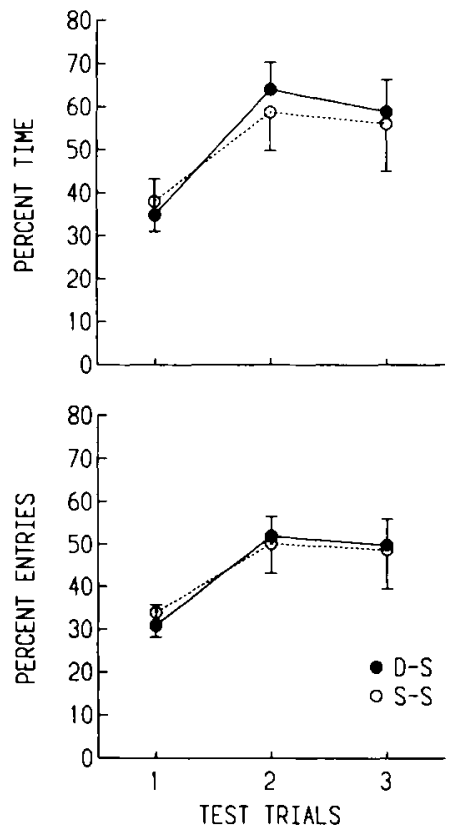

Figure 5. Mean percentages $( \pm S E M)$ of time spent in the open arms of the plus maze (upper graph) and entries into these arms (lower graph) by the groups in Experiment 3. Rats in both groups were injected with saline prior to each trial.

earlier phase had no impact on behavior in the latter. This agrees with studies of avoidance conditioning that have reported no effect of benzodiazepines upon extinction resulting from response prevention (Thyer et al., 1988). Apparently, the persisting benefit of benzodiazepine administration, such as that observed in Experiments 1 and 2, will appear only when exploration of the open arms is voluntary and not forced.

The animals in Group S-S differed from their counterparts in Experiments 1 and 2 in that the former received diazepam injections in their home cages on days when they were not placed in the maze. Though diazepam may have had some residual effect on these animals during the openarm exposures in Trials 1-5, we know that it could not have been as powerful as the temporally proximal (to the plus maze) effect experienced by Group D-S. The differences in bolus counts obtained from the two groups indicates this quite clearly. Nevertheless, the inclusion of a group that did not receive diazepam at any time would have provided useful information about the impact of extra-maze diazepam experience on plus-maze activity patterns.

\section{GENERAL DISCUSSION}

It is arguable that the decreased avoidance of the open arms of the plus maze that developed in the diazepamexperienced groups of Experiments 1 and 2 reflected a conditioned place preference. Diazepam induced an increased exploration of the open arms in these groups, and these areas could have become associated with the drug's 
reinforcing effects. When the drug was withheld during testing, the preference was retained. This explanation is unlikely for several reasons. Although diazepam will induce a place preference, the parameters in the present experiment probably precluded this effect (File, 1986). The animals were exposed to the maze for only $5 \mathrm{~min}$, and this exposure occurred well after the drug's effects had begun. File (1986) reported that she was unable to obtain reliable conditioning with less than four 45 -min training sessions, and her animals were injected $5 \mathrm{~min}$ prior to placement in a two-chambered test apparatus, so that the onset of drug action occurred in this place. Furthermore, those animals were confined to the nonpreferred side following diazepam administration. When we confined our animals to the initially nonpreferred open arms of the plus maze in Experiment 3, a procedure that should have facilitated the development of a place preference if one were to occur, no such effect was detected.

A simpler explanation assumes that diazepam reduces the aversiveness of the open arms and thereby facilitates exploration, allowing the animal to actively learn that these areas are "safe." This information is then retained, even in the absence of the drug. Learning about the safety of the open arms also appears to occur when exposure to the open arms is forced, as it was in Experiment 3. Note that both groups showed a preference for these areas (i.e., spent more than $50 \%$ of their time there) in their second and third exposures to the maze. Their percentages of time spent in the open arms and entries into them were very similar to those observed in the diazepamexperienced groups of Experiments 1 and 2. However, under this condition of forced exposure, diazepam did not facilitate learning, though it did reduce stress as indicated by the reduced bolus count.

Our statistical analysis of the first five plus-maze trials in Experiment 1 suggested that, although animals that received diazepam exhibited greater open-arm exploration relative to saline controls, the two groups did not differ in terms of the rate at which this exploration increased across trials (Figure 1). A potential explanation for this is suggested by the finding that exposure to a novel stimulus can trigger the release of an endogenous benzodiazepinereceptor ligand that may reduce the animal's response to the stimulus (Bodnoff, Suranyi-Cadotte, Quirion, \& Meaney, 1989). Diazepam, by also activating the same benzodiazepine receptor system, mimics the effect of habituation. If it is assumed that the effects of drug and habituation are merely additive, then the proportion of open-arm exploration should increase at the same rate as it does in animals that only receive saline.

In conclusion, our results indicate that diazepam does not cause anterograde amnesia under all circumstances. The differences between our experiments and those that found an impairment of habituation (Lister \& File, 1987; Platel \& Porsolt, 1982; Venault et al., 1986) are many, and include choice of species (mice vs. rats) and type of benzodiazepine (diazepam vs. chlordiazepoxide), as well as choice of procedure and apparatus. Despite these many potential reasons for the conflicting results, we suggest that a factor of substantial importance may be that the present experiments employed multiple exposures to the novel apparatus and repeated testing in the drug-free state, rather than just one habituation and one test trial. Several exposure sessions may serve to attenuate the effects of diazepaminduced amnesia. Furthermore, the use of at least two test trials can help to avoid erroneous conclusions that may be drawn when shifts from drug to nondrug states cause the sort of loss of habituation seen between Trials 5 and 6 in Experiment 1. Interestingly, Iwahara and Sakama (1972), who gave their rats four habituation trials, also noted such a disruption of habituation between their last habituation trial, when rats were given chlordiazepoxide, and their first test trial, when these animals were shifted to saline. This effect was substantially diminished a day later, during the next test session.

\section{REFERENCES}

BAUM, M. (1970). Extinction of avoidance responding through response prevention (flooding). Psychological Bulletin, 74, 276-284.

Bodnoff, S. R., Suranyi-Cadotte, B. E., Quirion, R., \& Meaney, M. J. (1989). Role of the central benzodiazepine receptor system in behavioral habituation to novelty. Behavioral Neuroscience, 103, 209-212.

Crawley, J. N. (1985). Exploratory behavior models of anxiety in mice. Neuroscience \& Biobehavioral Reviews, 9, 37-44.

Figler, M. H. (1973). The effects of chlordiazepoxide (Librium) on the intensity and habituation of agonistic behavior in male Siamese fighting fish. Psychopharmacologia, 33, 277-292.

FILE, S. E. (1985). What can be learned from the effects of benzodiazepines on exploratory behavior? Neuroscience \& Biobehavioral Reviews, 9, 45-54.

FILE, S. E. (1986). Aversive and appetitive properties of anxiogenic and anxiolytic agents. Behavioural Brain Research, 21, 189-194.

File, S. E., Johnston, A. L. (1987). Chronic treatment with imipramine does not reverse the effects of 3 anxiogenic compounds in a test of anxiety in the rat. Neuropsychobiology, 17, 187-192.

File, S. E., Pellow, S. (1988). Low and high doses of benzodiazepine receptor inverse agonists respectively improve and impair performance in passive avoidance but do not affect habituation. $B e$ havioural Brain Research, 30, 31-36.

Gorman, J. E., Dyak, J. D., \& Rid, L. D. (1979). Methods of deconditioning persisting avoidance: Diazepam as an adjunct to response prevention. Bulletin of the Psychonomic Society, 14, 46-48.

IwAHARA, S., \& SAKAMA, E. (1972). Effects of chlordiazepoxide upon habituation of open-field behavior in white rats. Psychopharmacologia, 27, 285-292.

Johnston, A. L. (1988). Can animal tests of anxiety detect panicpromoting agents? Human Psychopharmacology, 3, 149-152.

Johnston, A. L., \& File, S. E. (1989). Yohimbine's anxiogenic action: Evidence for noradrenergic and dopaminergic sites. Pharmacology, Biochemistry \& Behavior, 32, 151-156.

Kamano, D. K. (1972). Using drugs to modify the effect of response prevention on avoidance extinction. Behaviour Research \& Therapy, $10,367-370$.

LAdER, M. H. , WING, L. (1965). Comparative bioassay of chlordiazepoxide and amylobarbitone sodium therapies in patients with anxiety states using physiological and clinical measures. Journal of Neurology Neurosurgery \& Psychiatry, 28, 414-425.

LISTER, R. G. (1987). The use of a plus-maze to measure anxiety in the mouse. Psychopharmacology, 92, 180-185.

LISTER, R. G., \& FILE, S. E. (1987). The effect of chlordiazepoxide 
on the habituation of exploration: Interactions with the benzodiazepine antagonist RO 15-1788. Pharmacology, Biochemistry \& Behavior, 26, 631-634.

Nomikos, G. G., \& Spraki, C. (1988). Influence of oestrogen on spontaneous and diazepam-induced exploration of rats in an elevated plus maze. Neuropharmacology, 27, 691-696.

Overton, D. A. (1964). State-dependent or "dissociated" learning produced with pentobarbital. Journal of Comparative \& Physiological Psychology, 57, 3-12.

Pellow, S. (1986). Anxiolytic and anxiogenic drug effects in a novel test of anxiety: Are exploratory models of anxiety in rodents valid? Methods \& Findings in Experimental \& Clinical Pharmacology, 8, 557-565.

Pellow, S., Chopin, P., File, S. E., \& Briley, M. (1985). Validation of open:closed arm entries in an elevated plus-maze as a measure of anxiety in the rat. Journal of Neuroscience Methods, 14, 149-167.

Pellow, S., \& File, S. E. (1986). Anxiolytic and anxiogenic drug effects on exploratory activity in an elevated plus-maze: A novel test of anxiety in the rat. Pharmacology, Biochemistry \& Behavior, 24, 525-529.

Platel, A., Porsolt, R. D. (1982). Habituation of exploratory activity in mice: A screening test for memory-enhancing drugs. Psychopharmacology, 78, 346-352.
TAukuUs, H. K., Brake, L. D. (1988). Conditional drug interactions: Diazepam activity following diazepam-chlorpromazine pairings. Canadian Psychology, 29(2a), 773.

TAUKULIS, H. K., BRAKE, L. D. (1989). Therapeutic and hypothermic properties of diazepam altered by a diazepam-chlorpromazine association. Pharmacology, Biochemistry \& Behavior, 34, 1-6.

THIEBOT, M. H. (1985). Some evidence for amnesic-like effects of benzodiazepines in animals. Neuroscience \& Biobehavioral Reviews, 9, 95-100.

Thyer, B. A., BAUM, M., ReID, L. D. (1988). Exposure techniques in the reduction of fear: A comparative review of the procedure in animals and humans. Advances in Behavior Research \& Therapy, 10, 105-127.

Venault, P., Chapouthier, G., Prado de Carvalho, L., SimiAND, J., MOORE, M., DodD, R. H., Rossier, J. (1986). Benzodiazepine impairs and $\beta$-carboline enhances performance in learning and memory tasks. Nature, 321, 864-866.

(Manuscript received August 14, 1989; revision accepted for publication April 24, 1992.)

\section{News and Notes from NSF}

Most proposals to the National Science Foundation for research and related activities in animal learning and behavior are reviewed by the Advisory Panel for Animal Learning and Behavior. Current members of the Panel are Peter D. Balsam (Barnard College), Carol M. Berman (SUNY, Buffalo), Michael D. Breed (University of Colorado), Ruth E. Buskirk (University of Texas), Edmund J. Fantino (University of California, San Diego), Stewart H. Hulse (Johns Hopkins University), Alan C. Kamil (University of Nebraska), Patricia L. Schwagmeyer (University of Oklahoma), Judy A. Stamps (University of California, Davis), George W. Uetz (University of Cincinnati), and Ken Yasukawa (Beloit College).

The Advisory Panel for Animal Learning and Behavior wishes to maintain the highest methodological standards in observational as well as experimental research. Proposals for observational studies of behavior should describe how observers are trained, how interobserver reliability is assessed, and how observer bias is prevented or controlled.

Most proposals with major neurobiological aspects are reviewed by the following Neuroscience Programs. Each Program Director's name appears in parentheses:

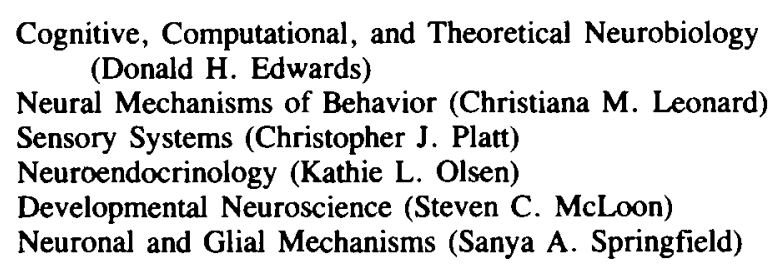

As a result of the recent reorganization of Biological, Behavioral, and Social Sciences at NSF, all of the Programs named above are in the Division of Integrative Biology and Neuroscience (IBN), one of four Divisions in the Directorate for Biological Sciences (BIO).

Target dates for submission of proposals to be reviewed by the Advisory Panel for Animal Learning and Behavior have changed. The new target dates are June 15 and December 15 each year. Neuroscience target dates remain January 15 and July 15. Proposals for Minority Research Initiation and Research in Undergraduate Institutions are reviewed by the disciplinary panels on the same schedule as other research proposals.

Such competitions as NSF Graduate Fellowships, Presidential Faculty Fellows, NSF Young Investigators, Research Planning Grants and Career Advancement Awards for Women Scientists, and the Special Competition for Basic Research in Conservation and Restoration Biology have specially-convened panels and annual deadlines for proposal submission.

For more information, call Fred Stollnitz, Program Director for Animal Behavior, at 202-357-7949, or any of the Neuroscience Program Directors at 202-357-7040. The IBN Fax number is 202-357-7846. 